\title{
Ecclesia, officium, ministerium: los modos de pensar la institucionalización eclesiástica en los concilios visigodos
}

\section{Ecclesia, Officium, Ministerium: the Ways of Conceiving Ecclesiastic Institutionalization in Visigothic Councils}

\author{
Eleonora Dell' Elicine \\ Universidad de Buenos Aires, \\ Universidad Nacional de General Sarmiento \\ eleonoradellelicine@gmail.com
}

El siguiente artículo explora qué se entiende por "Iglesia" en el reino visigodo de Toledo. Palabra polisémica por excelencia, con ella las fuentes visigodas dan nombre a cosas diferentes y - lo que genera más confusión - a varias cosas a la vez. A partir de un análisis del vocabulario conciliar, se advierte que a lo largo de los siglos vi y vir hubo modos diferentes de concebir la institucionalidad de la iglesia, pero finalmente en el concilio IV de Toledo y hasta la caída del reino se consolida un modelo de participación en los asuntos del reino que privilegia la integración por sobre la autonomización eclesiástica.

Palabras Clave: reino visigodo, iglesia, institucionalización

The following contribution explores what is meant by "Church" in the Visigoth kingdom of Toledo. Through this very preminent polysemic word, the Visigothic sources refer to different things and - what is utmost confusing- to various objects at the same time. Examining the conciliar vocabulary, we note that during the sixth and the seventh centuries there were different ways of considering the institutionalization of the Church. At the end, in the IVth. Council of Toledo up to the fall of the Kingdom, a model of participation in the Kingdom's affair that favoured integration over ecclesiastical autonomy became established.

KEYWORDS: visigothic kingdom, church, institutionalization

os historiadores coinciden ampliamente en que el concilio IV de Toledo, organizado por Isidoro de Sevilla en 633, resultó un punto clave en la conformación del reino visigodo católico. De esta manera 
analiza sus efectos Isabel Velázquez, la célebre editora de las aún más célebres pizarras, y una de las principales expertas en sociedad visigoda. Dice Velázquez:

[Como consecuencia de Toledo IV], el rey y la monarquía retuvieron su poder sobre todos los demás y su control sobre el regnum a través del juramento de lealtad comprometido por los otros [... ]. Una de las principales aspiraciones de la nobleza fue gradualmente alcanzada, mientras, al mismo tiempo, se reconocía su rol prominente en el reino. La iglesia adquiere un rol decisivo y determinante en la selección del monarca. De esta forma, la iglesia católica, cuyos representantes principales son tanto hispanorromanos como visigodos, se convierte en el tercer elemento del poder. ${ }^{1}$

Monarquía, nobleza, iglesia ... De estas tres grandes rúbricas, la que alcanza mayor consistencia y precisión durante el reino visigodo sin duda alguna es la monarquía. ${ }^{2}$ Acerca de la conformación de la nobleza, mucho nos enseña Velázquez en este mismo artículo. ${ }^{3}$ Resta por ver qué entendemos por iglesia, con el propósito principal de que este examen nos ayude a visualizar cómo se organiza el poder en el reino visigodo a partir del concilio IV de Toledo hasta la desaparición del reino.

\footnotetext{
1 "The king and the monarchy retained their power over all the others and their rule over the regnum by means of the oath of fealty sworn by the others. But there was a legal caveat: he must be chosen and could not, therefore, try to make the monarchy hereditary, ignoring the nobility's interests $[\ldots]$. One of the main aspirations of the nobility is gradually being accomodated while, at the same time, recognising its prominentrole within the kingdom $[\ldots]$. The Church takes on a decisive and determined role in the selection of the monarch. Thus, the Catholic church, whose main representatives are both Hispano-Roman and Visigothic, becomes the third element of power" (Velázquez, Pro patriae, 200 ss.). Sobre monarquía visigoda, más recientemente véase Martin, La geographie, "La reforme" y "L' innovation". También en esta línea, y específico sobre realeza visigoda, el trabajo de Valverde Castro, Ideología, simbolismo; Jiménez Garnica, "Sobre rex y regnum", Frighetto, "O problema da legitimidade".

2 "In the strict sense we might put foward the idea that the only clearly defined and regulated political institution in the history of the Visigoths was the monarchy, and the others that we referred to were other political instruments of the monarchy, or, regarding ists relations of power with the clergy and the aristocracy, mechanisms for limiting or controlling its power" (Díaz Martínez, "Visigothic political institutions", 347).

${ }^{3}$ Sobre aristocracia visigoda, también recientes véase Chavarría, "Poblamiento rural" y "Aristocracia tardoantigua"; García Moreno, "Etnía goda". Un enfoque arqueológico en Ripoll y Arce, "The transformation". Generales para el periodo, pero inteligentes e instructivos Wickham, "Society"; Framing; Le Jan, La société, Morsel, L'aristocratie.
} 


\section{ECCLESIA, OFFICIUM, MINISTERIUM. Desde el Concilio de Elvira hasta Sevilla II}

Como enseña Velázquez, las palabras resultan un indicador inmejorable para
problematizar las evidencias. ${ }^{4} \mathrm{El}$ método que utilizaremos es identificar bajo
qué sintagmas en el reino visigodo se designan realidades como "iglesia", "je-
rarquía eclesiástica", etc., tan claras para nosotros. ${ }^{5}$ El corpus con el que prin-
cipalmente vamos a trabajar son los concilios, dado que las formulaciones

4 "One thing is certain: that there exists a direct relationship between reality and the way in which it is recounted and transmitted and that, on occasions, one exerts a powerful influence on the other and vice versa. That is why the approach I intend to adopt in studying the topic is, in fact, to tackle those written sources-broadly described as literary-that 'talk' about Visigothic Hispania in the 6th-7th centuries, that deal with the gens Gothorum and their kings as well as the regnum they established $[. .$.$] . It is an issue that I regard as funda-$ mental, that is the issue of sources, not only because they are written in Latin (and Greek), from the Roman standpoint, but also because 'this is not only a question of criticism of our sources', but of the need to take into account contemporary authors' perception of events. Indeed, it is not simply a question of philological criticism of the sources but of something more fundamental because, without an analysis of both the form and content of the sources, treating each text in relation to others, it is not possible to interpret accurately the reality they convey. Moreover, the literary genres and type of documentation to which they belong must also be taken into consideration.

Council Proceedings and legislation, especially the Liber ludicum or Lex Visigothorum for the period we are studying, are texts of a legislative nature written in juridical language, in many cases with a long Roman tradition and using technical terms that are already standardised, but showing the evolution of some terms that reflect the contemporary reality about which they are legislating. On occasions, also, some were penned by the author himself, as we will see in the case of the Council of Toledo, or they were written following the models and literary references found in the great Christian authors such as Augustine, Jerome and Gregory the Great and included biblical quotations. These circumstances make these sources unique, both due to the way they were written and the particular language and specific terms used" (Velázquez, Pro patriae, 166).

${ }^{5}$ En 1975, un especialista en iglesia siríaca, Robert Murray, constataba: “[the syriac's] thought on the Church is expressed almost entirely through typology and imaginery, either tradicional or freely invented. What formal or systematic concerns they have are quite different from those of our western theological treatises. 'Ecclesiology' in particular, as a formal subject of study, is a late development even in the west [...]" (Symbols of Church, 1-2). A pesar del énfasis con que Murray lo propone, este modo de pensar a la iglesia durante los siglos altomedievales no es propio de los siríacos, sino común a la iglesia universal. Ver para ello De Lubac, Corpus mysticum; y Congar, La ecclesiologie; para un período posterior Boureau, L'événement, un panorama general de la iglesia en la Edad Media en Sánchez Herrero, Historia; a manera de ejemplo.

Medievalia 50, 2018, pp. 287-298 
jurídicas buscan de manera expresa la precisión conceptual. ${ }^{6}$ Comenzaremos por concilios anteriores al dominio visigótico, en la medida que estos heredan un vocabulario generado durante la dominación romana.

La palabra ecclesia en los concilios registra múltiples valores. En algunos cánones, se entiende como la asamblea local de fieles; ${ }^{7}$ en otros como edificio destinado al culto sagrado; ${ }^{8}$ en contextos por lo general referidos a herejías o a penas consensuadas como graves se alude a la iglesia universal; ${ }^{9} \mathrm{y}$ para terminar, frecuentemente hace referencia a dos o más de estos aspectos a la vez. ${ }^{10}$ No es casual que — a pesar de la búsqueda de precisión ya señalada para el género jurídico-, esta palabra revista en los concilios un valor polisémico. Se busca consolidar a la palabra en un plan calculadamente metonímico: más sencillamente, los concilios ponen en relación cosas distintas como si fuesen todo y partes de lo mismo. ${ }^{11}$

\footnotetext{
${ }^{6}$ Para profundizar el estudio de la legislación conciliar visigoda Orlandis y Ramos Lissón, Historia de los concilios; Orlandis, "La problemática”; Sánchez Herrero, "Concilios y sínodos"; Stocking, Bishops. Para un estudio de la dogmática y de los concilios generales resulta imprescindible el trabajo monumental de Charles Hefele, Histoire des concilies y también, más ligado a lo jurídico, el impresionante de Gabriel Le Bras, Histoire du droit.

7 “[...] Quoniam singuli coepimus in ecclesiis nostris facere diversa, et inde tant a scandala sunt, quae usque cisma perveniunt, si placet conmuni consilio decernimus quid ab omnibus episcopis in ordinandis clericis sit sequendum". Tol. I, introd. El concilio data según la cronología de Vives de 397- 400. Lo resaltado es nuestro.

8 "Ne picturae in ecdesia fiant: Placuit pinturas in ecclesia esse non debere, ne quod colitur et adoratur in parietibus depingatur". Elv. XXXVL El concilio data de 300-306. La relación entre la concepción de iglesia y el edificio de culto está magistralmente analizado en logna Prat, La maison.

${ }^{9}$ En relación con las herejías: "Haeretici si se transferre noluerint ad ecdesiam catholicam, ne ipsis carbólicas dandas puellas; sed ñeque iudaeis ñeque haereticis dare placuit, eo quod nulla possit esse societas fideli cum infidele. Si contra interdictum fecerit parentes, abstineri per quinquennium placet”. Elv. XVI.

10 "Ut si cuiuslibet ordinis clericus tardius ad ecdesiam fuit: Presbyster vel diaconus vel subdiaconus vel quilibet ecdesiae deputatus clericus, si intra civitatem fiaerit vel in loco in quo est ecdesia aut castelli aut vicus aut villae, ad ecdesiam ad sacrificium cotidianum non venerit, clericus non habeatur, si castigatus per satisfactionem veniam ab episcopo noluerit promereri". ToL I, V.

${ }^{11}$ El ejercicio metonímico registra una larga tradición en la iglesia; y deviene fundamentalmente del trabajo exegético. Así lo explica De Lubac: "U Ecriture est comme le monde: 'indéchiffrable dans sa plénitude et dans la multiplicité de ses sens. Forét profonde, au branchage innombrable, 'infinita sensuum silva': plus on s'engage, et plus on découvre qu'il est impossible de 1' explorer jusqu' au bout. Table dressée para la Sagesse, chargée de mets, oü l'insondable divinité du Sauveur s'ofFre elle-méme á tous en nourriture. Trésor du Saint-Esprit, dont la richesse est infinie comme lui" (De Lubac, Exégèse, 119-120).
}

Medievalia 50, 2018, pp. 287-298 
A diferencia de los autores posteriores a la reforma gregoriana, ${ }^{12}$ los concilios hispanos no utilizan el sintagma ecclesia para designar a los expertos en lo sagrado: para ello reservan la palabra clerus o su derivado clericus. Ya el primer concilio, el de Elvira, designa con este último nombre a quienes han recibido las órdenes sagradas y participan en la administración del sacramento, vale decir los episcopi, presbyteres et diacones. ${ }^{13}$ Progresivamente, esta lista se complejiza: en Toledo I, celebrado en 397 , se nombran subdiáconos, lectores y ostiarios. ${ }^{14} \mathrm{Al}$ conjunto de las funciones que cumplen se les denomina ministerium, officium publicum, clericalis officium o ministerium ecclesiasticum, ${ }^{15}$ empleándose este último sintagma como adjetivo que califica la función. El sustantivo ecclesia sigue utilizándose exclusivamente en los campos que ya hemos señalado.

Podemos claramente constatar entonces que a comienzos del siglo vi y agrupadas bajo rótulos políticos, se registran tareas distintas. En ese momento, $y$ al calor del fortalecimiento del poder obispal y del instrumento conciliar, cada uno de los cargos se precisa en torno a más criterios: la edad, la conducta, la vestimenta, el peinado, un rol específico en la liturgia, etc. ${ }^{16}$ Esto vuelve más explícito el acercamiento entre el cursus honorum civil y la promoción eclesiástica, en un momento donde las aristocracias provinciales hacen uso

${ }^{12}$ En tanto punto de inflexión fiindamental, gesto que inaugura una eclesiología diferente de la practicada en los siglos anteriores a ella, la reforma gregoriana arroja luz sobre los siglos que aquí nos interesan. La bibliografía sobre reforma es inmensa. En español, la compilación de Estella resulta fundamental (La reforma). Véase un panorama general actualizado en Sánchez Herrero, Historia.

13 "De clericis negocia et nundinas sectantibus: Episcopi, presbyteres et diaconeis in locis suis negotiandi causa non discedant, nec circumeuntes provincias quaestiosas mundinas sectentur. Sane ad victum sibi conquirendum aut filium aud libertum aut mercennarium aut amicum aut quemlibet mittant; et si voluerint negotiari, intra provincia negotientur" (Elv. XVIIII)

14 "Ut si diaconus, si defuncta uxore aliam duxerit, ostiarius fiat: Subdiaconus, autem defuncta uxore si aliam duxerit, et ab officio in quo ordinatus fuerat removeatur; et habeatur inter ostiarios vel inter lectores, ita ut evangelium et Apostolum non legat, proptereat ne qui ecdesiae servierit publicis officiis servire debeatur. Qui vero tertiam, quod nec dicendum aud audiendum est, acceperit, abstentus biennio, postrea inter laicos reconciliatus per poenitentiam communicet" (Tol. I, IV).

15 "Monaci a monasterio foras egredientes ne aliquod ministerium ecdesiasticum praesumant agere prohibemus, nisi forte cum abbatis imperio [...] (Tarrag. XI).

16 " [...] Utatur quisquis honoris sui concesso privilegio, quod propium sit ordinis presbyterii, non quod summi pontificatus est improbus minister assumat. Quisquis post hanc ammonitionem in huiuscenmodi rebus alquatenus fuerit deprehensus anathematis insolubili vinculo se noverit esse dampnandum [...]” (Tol. II. Amonestación del obispo Montano).

Medievalia 50, 2018, pp. 287-298 
creciente de la vía clerical. A cada uno de estos cargos a su turno se le designa como un ordo o un gradus, adoptando el lenguaje del derecho para definir el estatuto de una función. ${ }^{17}$

En el camino de la conceptualización de la jerarquía episcopal, el concilio II de Braga marca un punto de inflexión importante. Como sabemos, este sínodo fue convocado en 572 por Martín de Braga con el fin de organizar a la iglesia sueva, cuyo rey se había convertido unos años atrás. ${ }^{18}$ En este contexto, se emplea el sintagma ecclesiasticum ordinem para hacer referencia al conjunto de los ordenados. ${ }^{19} \mathrm{~A}$ través de este procedimiento metonímico, los suevos comienzan a pensar colectivamente al clero como un orden social, concepto romano que como sabemos designa a un grupo jurídicamente instituido con funciones, deberes y privilegios propios.

292 Vuelta al reino visigodo, el concilio II de Sevilla de 619 organizado también por Isidoro continúa formalmente con la línea del sínodo bracarense. En efecto, a las tareas consagradas se las designa como ministerium o como bonos ecclesiasticus ${ }^{20}$ mientras que un conglomerado de materias recibe el

17 A manera de ejemplo: "IMPPR GRAT(IANUS), VAL(EN) TINIANUS ETTHEOD(OSIUS) AAA. (AD OLEAR) CHUM P(RAEFECTUM) U(RB I): Nihil est tam iniuriosum in co(nser) vandis et custodiendis gradibus dignitatu $(\mathrm{m})$ quam usrupationis ambitio. Perit enim om(nis) praerogativa meritorum, si absque respectu (et con)templatione vel qualitate etiam provect(iones e) meritae custodiendi honoris locus praes(umitor) potius quam tenetur, ut aut potioribus eripia(tur) id quod est debitum, aut inferioribus prosit quod est debitum, aut inferioribus prosit q(uod) videtur indebitum”. DAT. IIII KAL. IAN.CONST(ANTINO) P(OLI) (MERO)BAUDE II ET SATURNINO CONSS” (C. Th. VI, 5,1).

18 Véase un panorama general en Pina Manique e Alburquerque, "Mapa da Galizia”, y especialmente Tranoy, La Galice. Para liturgia sueva Bragança, “A liturgia”; comunidades monásticas Orlandis, "Las congregaciones”.

19 "De neofitis: Neofitus qui nuper baptizatus fuerit iam aetate legitima, non continuo liceat eum ad ecclesiasticum ordinem promoved, quia oportet illum prius doceri quod posit docere, et multo tempore post babtismum probari, ut bene probatus veniat ad clerum secundum praeceptum Apostoli dicentis: 'Non neofitus, ne forte in superbiam elatus in indicium incidat et laqueum diaboli'. Si autem succedenti tempore in aliquo peccato gravi a duobus vel tribus fuerit devictus, depositus de gradu suo cesset a clero.Si quis contra hanc regulam facere presumserit, quasi contraries magni concilii proiciatur a clero' (Bracc. II, XXII).

20 "De presbyteris vel diaconibus ab uno episcopo non deponiendis: Sexta actione conperimus Fragitanum, Cordobensis ecclesiae presbyterem, a pontífice suo iniuste olim delectum et innocentem exilio condemnatum, quem rursus ordini suo restituentes id denuo adversus preasumptionem nostram decrevimus, ut iuxta priscorum patrum synodialem sententiam nullus nostrum sine concilii examine deiciendum quemlibet presbyterem vel diaconum audeat; nam multi sunt qui indiscussis potestate tyrannica non auctoritate canónica damnat, et sicut nonnullos gratiae favore sublimant ita quosdam odio invidiaque permoti humiliant et ad levem opiniones auram condemnat, quorum crimen non adprobant. Episcopus enim 
mismo adjetivo (ecclesiastica jura, ${ }^{21}$ ecclesiasticum ordinem, ${ }^{22}$ mores ecclesiasti$\mathrm{cis}^{23}$ etc.). Lo que advertimos es que una serie de competencias se recortan como propiamente eclesiásticas.

Hasta este punto, el repaso realizado se centraba en concilios provinciales o en sínodos extranjeros. Resultan formas de disciplinar el clero de una diócesis, formas que a lo sumo buscan establecerse como modelo de acción y referencia para otras jurisdicciones. El sínodo que finalmente logra instituir a la reunión obispal como órgano regular de un gobierno de alcance general

presbyteris ac ministris solus honorem dare potest, auferre solus non potest. Si enim hii qui in saeculo a dominis suis honores libertatis adepti sunt in servitutis nexo non revolvuntur nisi publice apud praetores tribunali foro fiaerint accusati, quanto magis hii qui divinis altaribus consecrati honore ecclesiastico decorantur? Quo profecto nec ab uno damnari nec uno indicante poterunt honoris sui privilegiis exui, sed praesentati synodiali iudicio, quod canon de illis praeceperit, definiri" (Sev. II, VI).

21 "De bigamis ad presbyerium vel diaconatum non promovendis: Quarta actione nuntiatum est nobis apud Astigitanam ecclesiam quasdam nuper ordinationes inlicitas extitisse, ita ut quidam viduarum mariti levitarum ministerio sacrarentur: quos quidem convenir a gradu suscepto inritum devocari nec ultra provehi ad diaconii ministerium qui contra divina atque ecclesiastica iura instituti repperiuntur" (Sev. II, IV).

22 "Ne presbyter diaconum aut presbyterem ordinare presumat: Quinto iudicio ad cognitionem nostram Aniani Egabriensis diaconi relata deductum est de quibusdam ipsius ecclesiae clericis, quorum dum unus ad presbyterum, duo ad levitarum ministerium sacrarentur, episcopus eorum oculorum detentus dolore, fertur manum suma super eos tantum posuisse et presbyter quidam illis contra ecclesiasticum ordinem benedictionem dedisse; qui licet propter tantam praesumtionis audaciam poterat adcusatus iudicio praesenti damnari, si adhuc in corpore positus non fuisset mortis vocatione praeventus; sed quia iam ille examini divino relictus humano iudicio accusare non potest, hii qui supersunt et ab eo non consecrationis titulum sed ingominiae potius eloquium perceperunt, ne sibi licentiam talis ultra usurpatio faciat, decrevimus ut gradu sacerdotalis vel levitici ordinis quem perverse adepti sunt deposit! aequo indicio abutantur. Tales enim mérito iudicati sunt removendi, quia inventi sunt constituti” (Sev. II, V).

23 "De oequonomis ne ex laicis constituantur: Nona actione didicimus quosdam ex nostro collegio contra mores ecclesiasticas laicos habere in rebus divinis constitutos oeconomos. Proinde pariter tractantes elegimus, ut unusquisque nostrum secundum Chalcidonensium patrum decreta ex proprio clero oeconomum sibi constituta. Indecorum est enim laicum vicarium esse episcopi et seculares in ecclesia iudicare: in uno enim eodemque officio non decet dispar professio. Quod etiam in lege divina prohibetur dicente Moyse: Non arabis in bove simul et asino; id est homines diversae professionis in officio uno non sociabis. Unde oportet nos et divinis libris et sanctorum patrum obedire praeceptis constituentes/ut hii qui in administrationibus ecclesiae pontificibus sociantur discrepare non debeant nec professione nec habitu: nam cohaerere et coniungi non posunt quipus et studia et vota diversa sunt. Si quis autem episcopus post haec ecclesiasticam rem aut laicali procuratione administrandam elegerit aut crediderit, vere ut contemtor canonum et fraudator ecclesiasticorum rerum non solum Christo de rebus pauperum iudicatur reus, sed etiam et concilio manebit obnoxius" (Sev. II, VIII).

Medievalia 50, 2018, pp. 287-298 
es, como decía Velázquez, el IV de Toledo. ¿Qué idea de iglesia patrocina este concilio fundamental? ¿En cuáles palabras de las heredadas se va a apoyar? El análisis de las opciones lexicales nos ayudará a entender mejor la idea de iglesia abierta por el programa de Isidoro.

\section{El cuarto Concilio de Toledo}

Catorce años después de Sevilla II, Isidoro impulsa un nuevo concilio. A juzgar por los resultados, el expediente de la referencia modélica ha tenido efectos escasos sobre el resto de las diócesis, e Isidoro se vuelca de lleno en un modelo centralista apoyado en el Estado. Este modelo impone por medios jurídicos un

294 idéntico esquema disciplinar, sacramental y de sustento material examinado para Sevilla II válido a partir de este punto a todo el ámbito del reino.

Atendiendo a los sintagmas, las fórmulas empleadas por Toledo IV resultan desconcertantemente conservadoras. No sólo en relación con lo alcanzado en el concilio segundo de Braga, sino también respecto al propio sínodo de Sevilla; se advierte una vuelta atrás en la formulación de un enunciado que articule en su conjunto a la jerarquía episcopal. En efecto, se alude a la ecclesia catholica, ${ }^{24}$ a la ecclesiastica consuetudo $o^{25}$ a las eclesiasticas mores ${ }^{26}$ al

24 “Tanta est quorundam cupiditas, ut quidam eam adpetentes iuxta quod ait Apostolus etiam a fide erraverint; multi quippe hucusque ex sacerdotibus atque laicis accipientes a iudaeis muñera perfidiam eorum patrocinio suo foverant, qui non inmérito ex corpore Anti Christi esse noscuntur, quia contra Christum faciunt. Quiquumque igitur deinceps episcopus sive clericus vel seculares illis contra fidem christianam suffragium vel muñere vel favore praesteterit, vere ut profanus et sacrilegus anathema effectus ab ecclesia catholica et regno Dei efficiatur extraneus, quia dignum est ut a corpore Christi separetur qui inimicis Christi patronus efficitur" (Tol. IV, LVIII).

25 "Post rectae fidei confessionem, quae in sancta Dei ecclesia praedicatur, placuit, ut omnes sacerdotes qui catholicae fidei unitate conplectimur, nicil ultra diversum aut dissonum in ecclesiasticis sacramentis agamus, ne qualibet nostra diversitas apud ignotos seu carnales schismatis errorem videatur ostendere, et multis existat in scandalum varietas ecclesiarum. Unus igitur ordo orando atque sallendi a nobis per omnem Spaniam atque Galliam conservetur, unus modus in missarum sollemnitatibus, unus in vespertinis matutinisque officis, nec diversa sit ultra in nobis ecclesiatica consuetudo qui una fide continemur et regno; hoc enim et an [ti] qui cánones decreverunt, ut unaquaeque provincia et psallendi et ministeriandi parem consuetudinem teneat" (Tol. IV, II).

26 "Nulla pene res disciplinae mores ab ecclesia Christi depulit quam sacerdotum neglegentiam, qui contemtis canonibus ad corrigendos eclessiasticos mores synodum facere neglegunt $[\ldots]$... (Tol. IV, III). 
gradus ecclesiasticus ${ }^{27}$ de un modo más homologable a los sínodos de la primera mitad del siglo vi que a los posteriores. ¿Qué ha sucedido con los obispos? ¿Se vieron arrebatados de modo colectivo por un súbito ataque de amnesia? ¿Cómo podemos explicar, en el marco de un concilio que inaugura nuevas prácticas, la elección de fórmulas tan prudentes?

Dos hipótesis concurrentes podemos arriesgar para responder a esta cuestión. Una de ellas es que esta estrategia conservadora se adapta al estado real de las fuerzas. En el concilio IV de Toledo, en efecto, Isidoro está buscando aunar los diferentes cleros provinciales en un solo clero nacional; y la imagen más plástica de los grados dispuestos jerárquicamente en una carrera ascendente permite mayor margen de negociación que un concepto más rígido que haga énfasis en la unidad del orden. Simultáneamente, si la unidad del estamento no descansa en las palabras, sí lo debe hacer en las prácticas, y a esto se encamina la batería de medidas tomadas para homogeneizar al clero en las dimensiones más dispares: en lo formativo, ${ }^{28}$ en el grado de acceso a las sagradas escrituras y conocimiento de la doctrina cristiana, ${ }^{29}$ en la

27 "Hii qui in discrimine constituti poenitentiam accipiunt nulla manifesta acelera confitentes sed tantum peccatores se praedicantes, huiusmodi si revaluerint possunt etiam pro forum probitate ad gradas edessiasticos pervenire; qui vero ita ponitentiam accipiunt ut aliquod mortale peccatum perpetrasse publice fatenatur, ad clerum vel honores eclesiásticos pervenire nullatenus possunt, quia se confessione propria notaverunt" (Tel. IV, LIIII).

28 "Prona est omnis aetas ab adolescentia in malum, nicil enim incertius quam vita adolescentium; ob hoc constituendum oportuit, ut si qui in clero púberes aut adulescentes existunt, omnes in uno conclavi atril conmorentur, ut lubricae aetatis annos non in luxuria sed in disciplinis ecclesiasticis agant deputari probatissimo seniori, quem et magistrum doctrinae et testem vitae habeant. Quod si aliqui ex his pupilli existunt sacerdotal! tutela foveantur, ut et vita eorum a criminibus intacta sit, et res ab iniuria improborum. Qui autem his preaeceptis resultaverint, monasteriis deputentur, ut vagantes animi et superbi severiori regula distrigantur" (Tol. IV, XIX).

29 "Ignorantia mater cunctorum errorum máxime in sacerdotibus Dei vitanda est, qui docendi officium in populis susceperunt: sacerdotes enim legere sancta scribtura admonet, Paulo apostolo dicente ad Timotheum: 'Intende lectioni, exhortationi, doctrinae, semper permane in his'. Sciant igitur sacerdotes scripturas sanctas et cánones, ut omne opus eorum in praedicatione et doctrina consistant, atque aedificent cunctos tam fidei scientia quam operum disciplina" (Tol. IV, XXV). 
canonización de ciertos libros, ${ }^{30}$ en la disciplina, ${ }^{31}$ en la uniformización de la liturgia, ${ }^{32}$ etc. Isidoro pretende articular un clero nacional que esté en paralelo con la administración civil, que guarda esa escala. Al igual que la administración civil y a diferencia con la iglesia gregoriana, el clero visigodo reconoce al rey como su cabeza rectora; mas sobrepasa a la administración civil por la sacralidad de sus funciones. Se trata de una clerecía incrustada en el cuerpo social, que debe garantizar con todos los medios humanos posibles el camino salvífico para esa comunidad de fieles. La gravedad de esta tarea los puede enfrentar, incluso, con el mismo rey.

El esquema de tres poderes propuesto por Velázquez resulta pertinente, dado que es válido pensar en tres fuerzas que acuerdan un programa, pero que se diferencian entre sí. Dos indicios vuelven sin embargo necesario regresar sobre este esquema. Uno más general: el modelo está sintomáticamente apegado al paradigma de los tres poderes del estado moderno. Otro más específico: no hay palabra en la situación para señalar esta realidad.

Lo que Toledo IV instituye es una forma de pensar y de habitar ese lazo social: en efecto, desde las palabras y desde las prácticas, esa sociedad se percibe a sí misma como una ecclesia, o aún más, como un pueblo elegido, como un nuevo Israel. En ese esquema, la clerecía participa junto al rey de la conducción de ese pueblo, en una postura que la sitúa paradójicamente por abajo y por encima de él. El término que resulta más difícil de domesticar es la aristocracia. La monarquía se la tendrá que ver con este problema.

30 "Apocalypsum librum multorum conciliorum auctoritas et synodica sanctorum praesulum Romanorum decreta loannis evangelistae esse praescribunt, et inter divinos libros recipiendum constituerunt: et quia plurimi sunt qui eius auctoritatem non recipiunt atque in ecclesiam Dei praedicare contemnunt, si quis eum deinceps aut non receperit aut a Pascha usque ad Pentecosten missarum tempore in ecclesia non praedicaverit, excomunicationis sententiam habebit” (Tol. IV, XVII).

${ }^{31}$ A modo ilustrativo: "Non aliter placuit, ut quemadmodum antestites ita presbyteres atque levitae quos forte infirmitas aut aetatis gravitas in conclavi episcopi manere non sinit, ut et idem in cellulis suis testes vitae habeant, vitamque suam sicut nomine ita et meritis teneant" (Tol. IV, XXIII).

${ }^{32}$ Un ejemplo: "Post rectae fidei confessionem, quae in sancta Dei ecclesia praedicatur, placuit, ut omnes sacerdotes qui catholicae fidei unitate conplectimur, nicil ultra diversum aut dissonum in ecclesiasticis sacramentis agamus, ne qualibet nostra diversitas apud ignotos seu carnales schismatis errorem videatur otendere, et multis existat in scandalum varietas ecclesiarum $[\ldots] "($ Tol. IV, 2). 


\section{BIBLIOGRAFÍA}

Boureau, Alain, L'événement sans fin. Récit et Christianisme au Moyen Âge, Paris: Les Belles Lettres, 1993.

Bragança, Joaquim, "A liturgia de Braga”, Hispania Sacra XVII, 33:4, 1964.

Bras, Gabriel Le, Histoire du droit et des institutions de l' Eglise en Occident, 3 vols., Paris: Sirey, 1955.

Chavarría, Alexandra, "Poblamiento rural en el territorium de Tarraco durante la Antigüedad Tardía”, Arqueología y Territorio Medieval, 8, 2001.

Chavarría, Alexandra, "Aristocracia tardoantigua y cristianización del territorio ¿otro mito historiográfico?”, Revista di Archeologia Cristiana, 82, 2006.

Código Teodosiano, ed. de Theodore Mommsen, Berlín, 1954.

Concilios visigodos e hispanorromanos, ed. de José Vives, Barcelona-Madrid: CSIC, 1963.

Congar, Yves, L' ecclesiologie du Haut Moyen Âge. De Saint Grégoire le Grand à la désunion entre Byzance et Rome, Paris: Èditions du Cerf, 1968.

Díaz Martínez, Pablo, "Visigothic political institutions", en Peter Heather (ed.), The visigoths from the migration period to the seventh century. An ethnographic perspective, London: The Boydell Press, 2003.

Frighetto, Renan, "O problema da legitimidade e a limitaçao do poder régio na Hispania visigoda: o reinado de Ervigio (680- 687)”, Gerion, 22, 2004.

García Moreno, Luis, “Etnía goda e Iglesia hispana”, Hispania Sacra, 54, 2002.

Hefele, Charles, Histoire des conciles, Paris: Letouzy et Ané, 1907.

Iogna Prat, Dominique, La maison Dieu: une histoire monumentale de l'Eglise au Moyen Âge (v. 800- v. 1200), Paris: Seuil, 2006.

Jan, Régine Le, La société du Haut Moyen Âge (Vie IX siécle), Paris: Armand Colin, 2003.

Jiménez Garnica, Ana, “Sobre rex y regnum. Problemas de terminología política durante el primer siglo de historia de los visigodos”, Pyrineae, 35:2, 2004.

$L$ a reforma gregoriana y su proyección en la cristiandad occidental. Siglos XI-XII, Pamplona: Gobierno de Navarra, 2006.

Lubac, Henri De, Corpus mysticum. L'eucharistie et l' église au Moyen Âge, Paris: Aubier, 1949.

Lubac, Henri De, Exégèse médiévale. Les quatre sens de l'écriture, 2 vols., Paris: Aubier, 1959.

Martin, CÉLIne, La geographie du pouvoir dans l'Espagne visgothique, Paris: Septentrión, 2003.

Martin, CÉLINe, "La réforme visigothique de la justice: les années Recceswinth", en Nilda Guglielmi y Adeline Rucquoi (eds.), Derecho y justicia: el poder en la Europa medieval, Buenos Aires: CONICET/CNRS, 2008.

Medievalia 50, 2018, pp. 287-298 
Martin, Céline, "L'innovation politique dans le royaume deTolède: le sacre du souverain”, en Élections et pouvoirs politiques au Moyen Âge et $16^{e}, 17^{e}$ siècles (colloque international 30 nov.-2 déc. 2006) [en prensa].

Morsel, Joseph, $L^{\prime}$ aristocratie médiévale. La domination sociale en Occident $\left(v^{e}-x v^{e}\right.$ siècle), Paris: Armand Colin, 2004.

Murray, Robert, Symbols of Church and Kingdom. A study in early Syriac tradition, Cambridge: George Press, 1975.

OrLANDIS, JosÉ, "Las congregaciones monásticas en la tradición suevo-gótica", Anuario de Estudios Medievales I, 1964.

OrLANDIS, José, "La problemática conciliar en el reino visigodo de Toledo", Anuario de Historia del Derecho Español, XLVIII, 1978.

Orlandis, José y Domingo Ramos Lissón, Historia de los concilios de la España 298 romana y visigoda, Pamplona: Eunsa, 1986.

Pina Manique e Alburquerque, José, “Mapa da Galiza sueva”, Bracara Augusta IX-X/ 1:4, 1958-1959.

Ripoll, Gisella y Javier Arce, "The transformation and end of Roman Villae in the west (fourth-seventh centuries): problems and perspectives", en Gian Brogiolo, Nancy Gauthier y Neil Christiel (eds.), Towns and their territories between Late Antiquity and the Early Middle Ages, Leiden-Boston- Köln: Brill, 2000.

SÁnchez Herrero, José, “Concilios y sínodos hispanos e historia de la Iglesia española”, Hispania, 175:2, 1990, 499-529.

SÁnchez Herrero, José, Historia de la Iglesia, t. II: La Edad Media, Madrid: Biblioteca de Autores Cristianos, 2005.

Stocking, Rachel, Bishops, Councils and Consensus in the Visigothic Kingdom, 589633, Ann Arbor: University of Michigan Press, 2003.

Tranoy, Alain, La Galice Romaine, Paris: Publications du Centre Pierre Paris, 1981.

Valverde Castro, María del Rosario, Ideología, simbolismo y ejercicio del poder real en la monarquía visigoda: un proceso de cambio, Salamanca: Universidad de Salamanca, 2000.

Velázquez, Isabel, Pro patriae gentisque Gothorvm statu, en Helmut Goetz, Jörg Jarnut y Walter Pohl (eds.), Regna and Gentes. The relationship between Late Antiquity and early Medieval peoples and kingdoms in the transformation of the Roman World, Leiden-Boston: Brill, 2003.

Wickнам, Chris, “Society”, en Rosemund Mckitterick (ed.), The early Middle Ages. Europe 400-1000, Oxford: Oxford University Press, 2001.

Wickham, Chris, Framing the Early Middle ages, Europe and the Mediterranean, 400800, Oxford: Oxford University Press, 2005. 